\title{
SLC7A11 regulated by NRF2 modulates esophageal squamous cell carcinoma radiosensitivity by inhibiting ferroptosis
}

Lei Feng ${ }^{1,2}$, Kaikai Zhao ${ }^{3}$, Liangchao Sun ${ }^{2}$, Xiaoyang Yin ${ }^{1,2}$, Junpeng Zhang ${ }^{1,2}$, Conghe Liu ${ }^{1,2}$ and Baosheng $\mathrm{Li}^{\mathrm{i}^{*}}$ (1)

\begin{abstract}
Background: Solute carrier family 7 member 11(SLC7A11) is a component of cysteine/glutamate transporter, which plays a key role in tumor growth; however, its underlying effect on radiosensitivity in esophageal squamous cell carcinoma (ESCC) remains unclear. This study aimed to clarify SLC7A11's expression and correlation with nuclear expression of nuclear factor erythroid-2 (NRF2)-associated radioresistance in ESCC.

Methods: We included 127 ESCC patients who received radical chemoradiotherapy. Immunohistochemical staining was used to detect SLC7A11 and NRF2 nuclear expression, and the relationship between clinicopathological characteristics and survival rates or therapy response were evaluated. Western blot, dual-reporter assays and Chromatin immunoprecipitation (ChIP)-sequencing were used to analyze their relationship in vitro. Their roles in radioresistance were then investigated through multiple validation steps.
\end{abstract}

Results: NRF2 nuclear expression and SLC7A11 expression were overexpressed in ESCC tissues and were positively correlated with one another. NRF2 nuclear expression was significantly associated with tumor length, lymph node metastasis, and TNM stage, while SLC7A11 expression was associated with lymph node metastasis. Patients with high NRF2 nuclear expression and SLC7A11 expression had significantly shorter overall and progression-free survival, and poor treatment response. The multivariate model showed that NRF2 nuclear expression and SLC7A11 expression, sex and tumor location are independent prognostic factors. In vitro analysis confirmed that hyperactivation of NRF2 induced SLC7A11 expression by directly binding to its promoter region, promoting radioresistance, reducing radiotherapy-induced lipid peroxidation levels, PTGS2 expression, and radiotherapy-related ferroptosis morphologic features.

Conclusion: Our study reveals a connection between high SLC7A11 expression and NRF2 nuclear expression in patients with ESCC that was related to worse survival and poorer therapy outcomes. SLC7A11-mediated ferroptosis inhibition induced NRF2-associated radioresistance, highlighting potential of NRF2/SLC7A11/ferroptosis axis as future therapeutic targets against therapy resistance biomarker.

Keywords: Esophageal squamous cell carcinoma, NRF2, SLC7A11, Ferroptosis, Prognosis

\footnotetext{
*Correspondence: bsli@sdfmu.edu.cn

${ }^{2}$ Department of Radiation Oncology, Shandong Cancer Hospital and Institute, Shandong First Medical University, Academy of Medical Sciences, Jinan 250117, China

Full list of author information is available at the end of the article
}

\section{Background}

Chemoradiotherapy (CRT) remains an important treatment strategy in esophageal squamous cell carcinoma (ESCC); however, high heterogeneity with unclear molecular classifications leads to various treatment outcomes 
$[1,2]$, and resistance to therapy limits therapy efficacy to a large extent. Therefore, there is an urgent need to identify accurate biomarkers that predict and improve CRT response.

The cystine/glutamate antiporter SLC7A11 (or xCT) imports cystine for glutathione biosynthesis and antioxidant defense and is overexpressed in a variety of human cancers [3]. Recently, SLC7A11 has been found to play an important role in tumor growth, progression, and metastasis in various types of cancer including pancreatic tumors [4], glioblastomas [5], breast cancer [6], esophageal tumors [7], and ovarian cancer [8]. Overexpression of SLC7A11 suppresses ferroptosis $[9,10]-\mathrm{a}$ form of regulated cell death induced by excessive lipid peroxidation-correlating with better radiotherapy response and longer survival in cancer patients $[11,12]$.

NRF2 is an important transcription factor that regulates cellular antioxidant response [13] and is associated with radioresistance as illustrated in numerous studies [14-16]. However, the exact molecular mechanism of NRF2-mediated radioresistance remains unclear. Normally, low cellular NRF2 concentrations are maintained by proteasomal degradation through a Keap1-Cul3dependent mechanism [17]. Oxidative stress or similar circumstances, function to stabilize and activate NRF2 in the cell nucleus [18], regulating downstream gene transcription (including SLC7A11); this amplifies glutamate secretion, thereby affecting the tumor microenvironment [19]. NRF2 activation in cancer cells could prevent ferroptosis [13]. Still, whether NRF2-induced radioresistance relates to SLC7A11-mediated ferroptosis regarding ESCC has not been elucidated; additionally, the interaction between hyperactive NRF2 nuclear expression and SLC7A11 on the efficacy of CRT in ESCC patients remains unclear.

We aimed to evaluate NRF2 nuclear expression and SLC7A11 expression using immunohistochemistry (IHC) to determine their prognostic and therapy effects in ESCC patients who received radical CRT. This study is the first to assess the prognostic effects and radiotherapy (RT) response of and SLC7A11 expression in patients with ESCC who underwent radical CRT. Additionally, it provides a potential means of improving ESCC radiosensitivity by targeting the NRF2/SLC7A11/ferroptosis pathway.

\section{Materials and methods \\ Patients}

This study was approved by the Ethics Committee of Shandong Cancer Hospital and Institute (approval number: SDTHEC201803); the requirement of informed consent was waived due to the retrospective nature of the study. One hundred twenty-seven patients with ESCC who underwent CRT at our hospital between January 2014 and June 2019 were enrolled in this study. Eligibility criteria were pathology-confirmed ESCC, a Karnofsky performance status score $\geq 70, \geq 4$ chemotherapy (CT) cycles, and an RT dose $\geq 45$ Gy. Exclusion criteria were a history of other malignancies, having received RT only, and incomplete records. Patients' clinicopathological data were collected from their medical records, and the TNM classification was used to determine the tumor stage. Patients were followed up every 3 months posttreatment for the first year, and every 6 months thereafter. The last follow-up time was January 2021.

\section{IHC staining}

Samples obtained via pathological biopsy were routinely fixed in $10 \%$ neutral buffered formalin, cut into $3-4 \mu \mathrm{m}$ sections, and dried for $1 \mathrm{~h}$ at $65^{\circ} \mathrm{C}$; sections were deparaffinized with xylene and rehydrated with a graded ethanol series. Antigens were retrieved by heating the samples in EDTA ( $\mathrm{pH}$ 8.0) for $15-20 \mathrm{~min}$ at $95{ }^{\circ} \mathrm{C}$. Endogenous peroxidases were blocked by incubating sections in $3 \%$ hydrogen peroxide for $15 \mathrm{~min}$. Protein blocking was performed for $15 \mathrm{~min}$ and then incubated with anti-NRF2 (1:250 dilution, ab62352, Abcam, UK) and anti-SLC7A11 (1:200 dilution, ab37185, Abcam) primary antibodies overnight at $4{ }^{\circ} \mathrm{C}$; negative controls were incubated with phosphate buffered solution (PBS) instead of the primary antibodies. On day two, the slides were reheated at room temperature for $1 \mathrm{~h}$, washed 3 times with PBS, 5 min each time, and treated with a Novolink polymer for 10-15 min. Then, appropriate amount of biotin-labelled goat anti-rabbit IgG polymer was dropped, the slides were incubated at room temperature for $10-15 \mathrm{~min}$, washed 3 times with PBS; then appropriate amount of horseradase-labelled streptomycin working solution was dropped for 10-15 min, and washed 3 times with PBS. A working solution was prepared (1:20 DAB chromogen in DAB substrate buffer; Novolink) and applied for $3 \mathrm{~min}$; the slides were then counterstained with haematoxylin (Novolink) for $2 \mathrm{~min}$ and dehydrated. All the images were viewed using a BX53 fluorescence microscope (Olympus Corporation, Tokyo, Japan).

\section{Immunostaining scoring}

Five microscopic fields of the tumor tissues (original magnification, $\times 400$ ) were randomly selected. Two independent investigators, blinded to all immunohistochemical outcomes, adopted a semiquantitative system-considering staining intensity and proportion-to score all the specimens; NRF2 expression was thus semi-quantitatively assessed according to staining intensity in the cancer cells. Reactions were divided into four categories: negative $(0,0 \%)$, low $(1+, 1-33 \%)$, moderate 
$(2+, 34-66 \%)$, and high (3+, 67-100\%) [20]; expression was observed in either the nucleus or cytoplasm, with an intensity $\geq 1$ (Fig. 1). Because NRF2 is an important transcription factor, it usually plays a mechanistic role in the nucleus. Therefore, we focused on the assessment of tumor nuclear expression. Staining of 0 or $1+$ was defined as negative, and $2+$ or $3+$ was defined as positive.

SLC7A11 was mainly expressed on the membrane; it was divided into negative staining (same color as the background), low staining (slightly stronger color than the background), moderate staining (markedly stronger color than the background), and high staining; these were scored as $0,1,2$, and 3, respectively. According to the percentage of the positive cells in the field, $<10 \%$, $10-25 \%, 26-75 \%$ and $>76 \%$ were scored as negative (0), low $(1+)$, moderate $(2+)$, and high $(3+)$ staining, respectively. These two values were subsequently combined to determine the protein expression of each group (a final score of $0-2$ represented negative '-' staining, while 3-7 represented positive ' + ' staining) [21].

\section{Cell lines and lentivirus infection}

The human ESCC cell lines KYSE 30, KYSE 150, KYSE450, KYSE410, KYSE510, and TE-1 were obtained from the Key Laboratory of Shandong Cancer Hospital and Institute. The cells were maintained in DMEM supplemented with $10 \%$ FBS and 1\% penicillin-streptomycin antibiotics (Gibco Laboratories; Thermo Fisher Scientific, Massachusetts, USA) at $37{ }^{\circ} \mathrm{C}$ in a $5 \% \mathrm{CO}_{2}$ incubator (Galaxy 170R, Eppendorf, Hamburg, Germany). To overexpress NRF2, KYSE 150 and KYSE 30 cells were seeded in six-well plates; once $30-40 \%$ confluence was reached, lentiviruses carrying NRF2 (NM_006164.5; GenePharma Co., Ltd, Shanghai, China) were added. After $72 \mathrm{~h}$ of transduction, positively transduced cells were selected, using puromycin (NeoFroxx, Einhausen, Germany), for at least 3 days. The SLC7A11 siRNA target sequence was 5'-CTGGAGTTATGCAGCTAAT-3'. For transfection, cells were plated at a density of $1 \times 10^{5}$ cells/well in six-well plates; after $24 \mathrm{~h}$, all siRNA were transfected with siRNA-mate transfection reagent (GenePharma Co., Ltd.), and transfection was confirmed.

\section{Western blot assay and antibodies}

Cells were lysed in lysis buffer (Beyotime Biotechnology, Nanjing, China) containing protease inhibitor cocktails. Nuclear protein extraction and BCA Protein Assay Kits (Beyotime Biotechnology) were used for extraction and to determine protein concentrations, respectively. SDSPAGE (10\%) and Western immunoblotting were performed according to standard procedures; proteins were detected with antibodies recognizing SLC7A11 (1:1000 dilution, ab175186; Abcam), NRF2 (1:500 dilution, ab62352; Abcam) and GAPDH (1:2000 dilution, \#5174; Cell Signaling Technology, Massachusetts, USA).

\section{Quantitative-PCR (qPCR)}

Total RNA was extracted from the ESCC cell lines using the RNAsimple Total RNA Kit (TIANGEN, Beijing, China). For cDNA synthesis, 500 ng of total RNA was reverse transcribed in $10 \mu \mathrm{l}$ reaction volume using the PrimeScriptTM RT Master Mix (Perfect Real Time) (Takara Bio Inc., Shiga, Japan). After amplification, the resulting cDNA was subjected to qPCR using TB Green

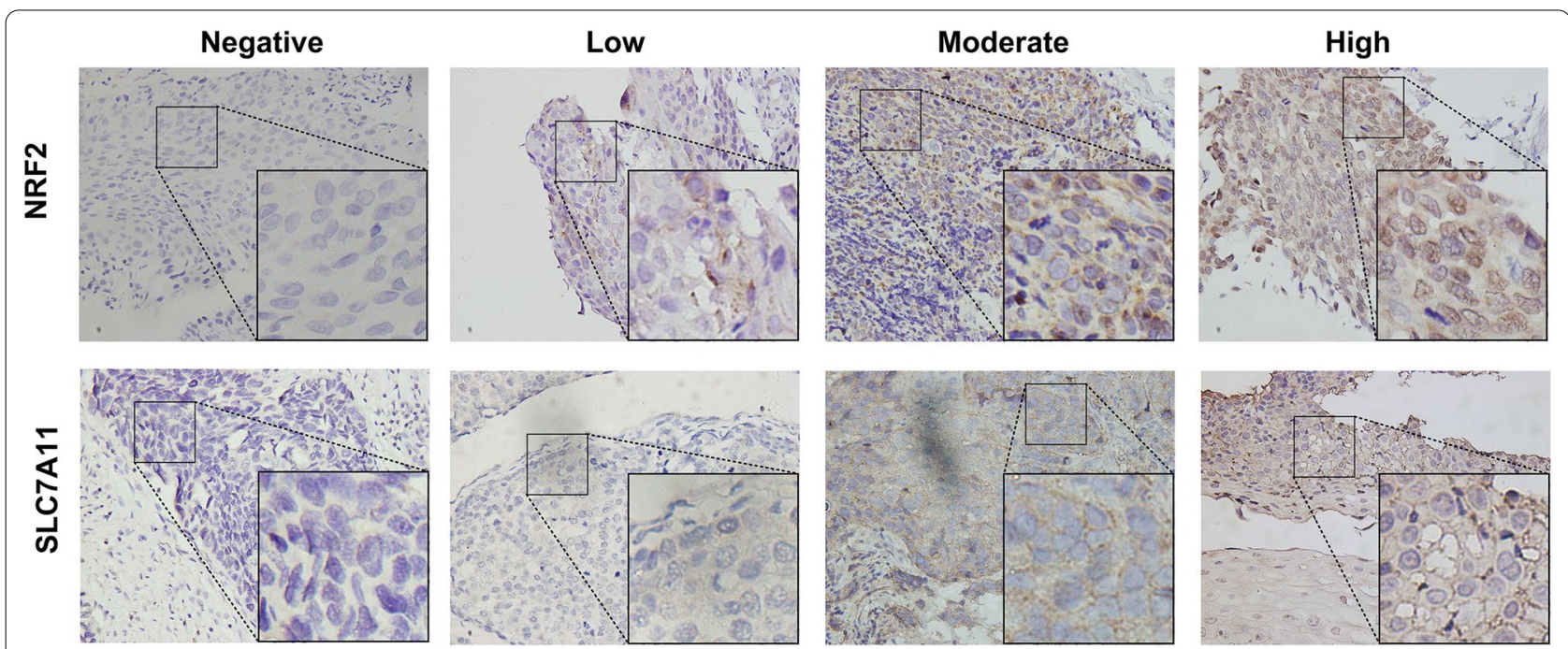

Fig. 1 NRF2 and SLC7A11 expression in ESCC tissues. Representative images for negative, low, moderate, and high NRF2 expression; negative, low, moderate, and high SLC7A11 expression ( $\times 400$ magnification). ESCC, esophageal squamous cell carcinoma 
Premix Ex Taq II (Tli RNaseH Plus, Takara Bio Inc.); GAPDH was selected as the housekeeping gene. The expression level of each gene was calculated via the $2^{-}$ $\Delta \Delta \mathrm{Ct}$ method. The qPCR primers included: NRF2, $5^{\prime}-\mathrm{TCC}$ AAGTCCAGAAGCCAAACTGAC-3' and 5'-GGAGAG GATGCTGCTGAAGGAATC-3'; SLC7A11, 5' -TTACCA GCTTTTGTACGAGTCT-3' and 5'-GTGAGCTTGCAA AAGGTTAAGA-3'; and GAPDH, 5'-GACCCCTTCATT GACCTCAAC- $3^{\prime}$ and $5^{\prime}$-CTTCTCCATGGTGGTGAA GA-3'.

\section{Cell proliferation assay and clonogenic survival assay}

Cell viability was measured with the use of the CCK- 8 assay (Bioss, Beijing, China). A 96-well plate was seeded with 2000 cells/well and cultured at $37{ }^{\circ} \mathrm{C}$ with $5 \% \mathrm{CO}_{2}$. The cells were pre-treated with $5 \mu$ MFerrostatin-1 (Cat\#SML0583, Sigma-Aldrich, Missouri, USA) or DMSO (Beijing Solarbio Science \& Technology Co., Ltd., Beijing, China) for $24 \mathrm{~h}$ before RT. RT was conducted with a cabinet irradiator ( $\mathrm{Rad}$ Source Technologies, Georgia, USA) at doses of $6 \mathrm{~Gy}$, and a dose rate of $165 \mathrm{MU} / \mathrm{min}$. Cell viability was determined following the manufacturer's instructions, and the optical density (OD) value was measured at $450 \mathrm{~nm}$ using a microplate reader (SpectraMax; Molecular Devices, California, USA). In addition, the blank background group-wells with only DMEM medium was set up to eliminate the OD value of medium. The calculation formula for the cell proliferation rate is: cell proliferation rate $(\%)=(\mathrm{OD}$ treatment group-OD Blank)/(OD control group-OD Blank) $\times 100 \%$ [22]. Cell survival following radiation exposure was defined as the cell's ability to maintain clonogenic capacity and subsequently form colonies. Cells were counted and seeded in six-well plates at 500 cells/ well. After $24 \mathrm{~h}$ pre-treatment with $5 \mu \mathrm{M}$ Ferrostatin-1, the cells were exposed to the indicated doses (0-6 Gy) of $\mathrm{RT}$ and incubated at $37{ }^{\circ} \mathrm{C}$ for $12-14$ days. Colonies were stained with crystal violet, manually counted, and those consisting of $\geq 50$ cells were scored. All experiments were performed in triplicate.

Reactive oxygen species (ROS) and lipid peroxidation assay ROS and lipid peroxidation levels were measured as previously described [23, 24]. Cells were seeded in six-well plates $24 \mathrm{~h}$ prior to RT and incubated for another $24 \mathrm{~h}$; $4 \mu \mathrm{M}$ CM-H2DCFDA (ThermoFisher, C6827, USA) for the total ROS measurements or $5 \mu \mathrm{M}$ BODIPY 581/591 C11 dye (Invitrogen, D3861, USA) for measurements of the lipid peroxidation levels was added per well, along with fresh medium. After incubation (30 min), cells were washed with PBS, trypsinized to a cell suspension with Trypsin-EDTA solution (Biosharp, Shanghai, China), and analyzed via flow cytometry (FACSCalibur; BD Biosciences, New Jersey, USA).

\section{Transmission electron microscopy (TEM)}

TEM analyses were performed as previously described [24]. Cells were collected by gently scraping and fixing samples with $4 \%$ paraformaldehyde (PFA) (Bioss, Beijing, China) for at least $2 \mathrm{~h}$ at $4{ }^{\circ} \mathrm{C}$, and dehydrated, at increasing concentrations of ethanol and propylene oxide after fixing with $0.1 \%$ osmium tetroxide. We then embedded the samples by cutting them into ultra-thin sections and staining them with $3 \%$ uranyl acetate and lead citrate; these were examined under the microscope (HT-7800; Hitachi Medical Corporation, Tokyo Japan).

\section{Dual-reporter assays}

Transient transfection of $5 \times 10^{4} 293 \mathrm{~T}$ cells with reporter luciferase plasmids and co-transfected plasmids (Shanghai GeneChem Co., Ltd., Shanghai, China) was performed in 24-well culture plates using the Lipofectamine 3000 Transfection Reagent (Invitrogen, Themo Fisher Scientific) as previously described [25]. Cells were harvested $48 \mathrm{~h}$ after transfection, and luciferase activities were measured using the Dual-Glo Luciferase Assay System (Promega Corporation, Wisconsin, USA). Transfection and reporter assays were performed in duplicate, and independently repeated at least three times.

\section{ChIP-sequencing analysis}

As were used in previous studies [26], KYSE 150 cells $\left(>1 \times 10^{7}\right)$ were crosslinked with $1 \%$ formaldehyde for $10 \mathrm{~min}$ at room temperature and quenched with $0.125 \mathrm{~mol} / \mathrm{L}$ glycine for $5 \mathrm{~min}$. In brief, nuclei were isolated by dounce homogenization and subjected to sonication to generate 200-1000 bp fragments in length, a portion of it was used to be the input DNA. The ProteinDNA complexes were immunoprecipitated with protein A + G magnetic beads coupled to the anti-NRF2(Cell Signaling Technology, 12721, America). ChIP DNA then were obtained by reverse cross-linking with proteinase $\mathrm{K}$ and phenol chloroform. ChIP DNA libraries were created in the following six successive steps: end repair (NEB No. E6050S); dA tailing (NEB No. E6053S); adaptor ligation (NEB No.E7445S); size selection (Beckman, Agencourt AMPure XP); PCR amplification (NEB No. $\mathrm{M} 0544 \mathrm{~S}$ ) using a PCR program (at $98{ }^{\circ} \mathrm{C}$ for $1 \mathrm{~min}, 12$ cycles of $98{ }^{\circ} \mathrm{C}$ for $10 \mathrm{~s}, 62{ }^{\circ} \mathrm{C}$ for $1 \mathrm{~min}, 72{ }^{\circ} \mathrm{C}$ for $1 \mathrm{~min}$ ); and finally extended to $72{ }^{\circ} \mathrm{C}$ for $5 \mathrm{~min}$. The constructed libraries were cleaned with Ampure beads and sequenced on Illumina HiSeq Xten system. Raw reads were filtered to obtain high quality clean reads by removing sequencing adapters, short reads (length $<50 \mathrm{bp}$ ) and low-quality reads using Cutadapt (v1.9.1) and Trimmomatic23 
(v0.35). Next, FastQC was used to ensure high reads quality. The clean reads were mapped to the reference Homo_sapiens_assembly38 (hg38) using the Bowtie2 (v2.2.6) software. Peak detection was performed using the MACS (v2.1.1) peak finding algorithm with 0.01 set as the $\mathrm{P}$ value cut-off. Annotation of peak sites to gene features was performed using the ChIPseeker R package.

\section{Statistical analysis}

Clinicopathological features were reported in frequencies. Chi-squared and Fisher's exact tests were used for categorical variables, and survival analysis was performed using the Kaplan-Meier method. Univariate and multivariate Cox regression hazards models were used to evaluate survival risk factors, and variables $(p<0.2)$ from univariate analysis were subject to multivariate analysis. An unpaired two-sided Student's t-test was used to compare groups at each time point, after ensuring that variances were similar between groups. Data are presented as the mean \pm SEM for three or more independent biological replicates. Statistical analyses were conducted using IBM SPSS Statistics for Windows, version 22.0 (IBM, Armonk, New York, USA) and GraphPad Prism 8.0 (GraphPad Software Inc., California, USA); $p<0.05$ was considered statistically significant.

\section{Results}

\section{Clinicopathological characteristics}

The clinicopathological characteristics of the study cohort $(n=127)$ are summarized in Table 1. Patient age ranged from 43-84 (median: 66) years; 34 patients received RT alone, 93 received CRT, and 97 received an RT dose $>54$ Gy. Patients who received CRT were treated with 4 to 6 courses of cisplatin-based CT (combined with paclitaxel/ docetaxel). Eighty (63.0\%) patients were men, $61(48.0 \%)$ patients smoked, and 47 (37\%) patients had a history of alcohol abuse. The tumor length ranged from $2.0-12.0 \mathrm{~cm}$ (median: $5 \mathrm{~cm}$ ). The number of patients with TNM stages T1-T4 was $3,19,77$ and 28 , respectively. The number of patients staged N0-3 was $26,79,19,3$, respectively, M0-1 was 114 and 13, respectively, and TNM stages I-IV were $1,30,59$, and 37 , respectively.

\section{NRF2 and SLC7A11 overexpression in ESCC tissues}

IHC was performed to detect NRF2 nuclear expression (representing NRF2 activation) and SLC7A11 expression. NRF2 and SLC7A11 were positively expressed in $61(48.0 \%)$ and $62(48.8 \%)$ patients, respectively (Fig. 1; Table 3). Additionally, we collected 62 pairs of tumor and matched adjacent normal tissue samples in 62 patients who had undergone radical esophagectomy. NRF2 was positively expressed in $37.1 \%$ (23/62) of the tumor samples, compared with $17.7 \%$ of the matched adjacent tissue
Table 1 The clinical characteristics of 127 ESCC patients

\begin{tabular}{|c|c|}
\hline Variables & ESCC, n (\%) \\
\hline \multicolumn{2}{|l|}{ Sex } \\
\hline Male & $80(63.0)$ \\
\hline Female & $47(27.0)$ \\
\hline \multicolumn{2}{|l|}{ Age } \\
\hline Range (Median) & $66(43-84)$ \\
\hline \multicolumn{2}{|l|}{ Alcohol abuse } \\
\hline Yes & $47(37)$ \\
\hline No & $80(63)$ \\
\hline \multicolumn{2}{|l|}{ Smoking } \\
\hline Yes & $61(48)$ \\
\hline No & $66(52)$ \\
\hline \multicolumn{2}{|c|}{ Location of primary tumor } \\
\hline Upper or middle & $56(44.1)$ \\
\hline Lower & $71(55.9)$ \\
\hline \multicolumn{2}{|l|}{ Length, cm } \\
\hline Range (Median) & $5(2-12)$ \\
\hline \multicolumn{2}{|l|}{ T stage } \\
\hline $\mathrm{T} 1-2$ & $22(17.3)$ \\
\hline T3-4 & $105(82.7)$ \\
\hline \multicolumn{2}{|c|}{ Lymph node metastasis } \\
\hline No-1 & $105(82.7)$ \\
\hline N2-3 & $22(17.3)$ \\
\hline \multicolumn{2}{|l|}{ Metastasis } \\
\hline MO & $114(89.8)$ \\
\hline M1 & $13(10.2)$ \\
\hline \multicolumn{2}{|l|}{ TNM stage } \\
\hline$|-| \mid$ & $31(24.4)$ \\
\hline III-IV & $96(75.6)$ \\
\hline \multicolumn{2}{|l|}{ Therapy method } \\
\hline RT & $34(26.8)$ \\
\hline CRT & $93(73.2)$ \\
\hline \multicolumn{2}{|l|}{ RT dose (Gy) } \\
\hline$>54$ & $97(76.4)$ \\
\hline$\leq 54$ & $30(23.6)$ \\
\hline
\end{tabular}

samples $(11 / 62)(p=0.016)$. Similarly, SLC7A11 expression was higher in tumor tissues than in matched adjacent normal tissues ( 56.5 versus $14.5 \%, p<0.001$; Table 2; Fig. 2a, b). These results imply that nuclear expression of nuclear NRF2 and SLC7A11 are overexpressed in ESCC tumor tissues.

\section{NRF2 nuclear and SLC7A11 expression correlates with the clinicopathological characteristics of ESCC}

Nuclear expression status of NRF2 and SLC7A11 and the clinical characteristic of patients with ESCC are summarized in Table 3. NRF2 nuclear expression had no significant correlation with age, sex, alcohol 
Table 2 Nuclear NRF2 and SLC7A11 expression in ESCC and matched adjacent tissues $(n=62)$

\begin{tabular}{llll}
\hline Variables & $\begin{array}{l}\text { Cancer } \\
\text { tissues, } \mathbf{n}(\%)\end{array}$ & $\begin{array}{l}\text { Matched adjacent } \\
\text { tissues, } \mathbf{n}(\%)\end{array}$ & P-value \\
\hline NRF2 & & & 0.016 \\
Low or no & $39(62.9)$ & $51(82.3)$ & \\
$\quad$ Moderate or high & $23(37.1)$ & $11(17.7)$ & $<0.001$ \\
SLC7A11 & & & \\
Negative & $27(43.5)$ & $53(85.5)$ & \\
Positive & $35(56.5)$ & $9(14.5)$ & \\
\hline
\end{tabular}

abuse and smoking history, tumor location, $\mathrm{T}$ stage, or lymph node metastases status. However, it was significantly higher in patients with tumors length $\geq 4 \mathrm{~cm}$ $(p=0.035)$ and in patients with later TNM stage $(p=0.015)$. While patients who developed lymph node metastases had significantly higher nuclear expression of NRF2 expression than those with no evidence of metastasis $(p=0.037)$, there was no significant correlation between distant metastasis and nuclear expression of NRF2 $(p=0.057)$.
The proportion of SLC7A11-positive patients tended towards high lymph node metastasis rates. Other covariates (age, sex, alcohol, smoking, location, tumor length, and TNM stage) were not significantly associated with SLC7A11 expression, while nuclear expression of NRF2 positively correlated with SLC7A11 $(p<0.001$, Fig. 3a). These results indicate that NRF2 nuclear expression and SLC7A11 expression are associated with tumor progression and metastasis in ESCC. As we mentioned earlier, the entry of NRF2 into the nucleus activates the NRF2 pathway while escaping ubiquitination and degradation. To validate the relationship between NRF2 and SLC7 A11, we conducted further explorations in vitro. Just as we expected, western immunoblotting analysis revealed that NRF2, expressed in six ESCC cell lines, positively correlated with SLC7A11 expression levels (Fig. 3b). Then, we determined whether there is a binding region between transcription factor NRF2 and SLC7A11 promoter using the JASPAR database [27] (http://jaspar.genereg.net/). Strikingly, the results suggested that SLC7A11 was a potential target of NRF2 (Fig. 3c). The dual-luciferase reporter assay showed that hyperactive NRF2 induced SLC7A11 expression by directly binding to its promoter
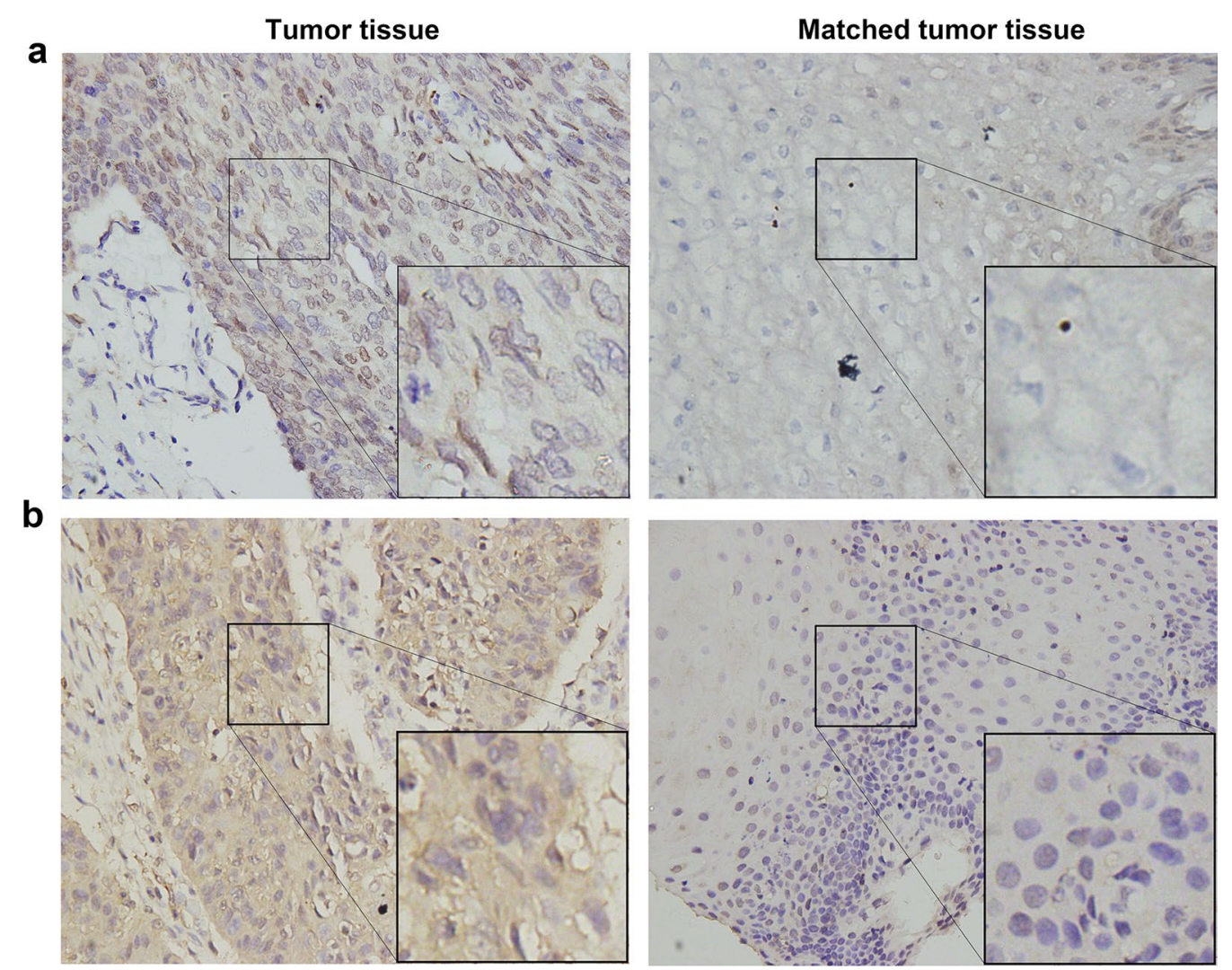

Fig. 2 NRF2 nuclear and SLC7A11 expression in ESCC tumor and matched adjacent normal tissues. Representative images of nuclear NRF2 (a) and SLC7A11 (b) expression in tumor and matched adjacent normal tissues ( $\times 400$ magnification) 
Table 3 Characteristics of patients with ESCC $(n=127)$

\begin{tabular}{|c|c|c|c|c|c|c|}
\hline \multirow[t]{2}{*}{ Variables } & \multicolumn{3}{|l|}{ NRF2 } & \multicolumn{3}{|l|}{ SLC7A11 } \\
\hline & Low (66) & High (61) & P-value & Negative (65) & Positive (62) & $P$-value \\
\hline Sex & & & 0.189 & & & 0.728 \\
\hline Male & 38 & 42 & & 40 & 40 & \\
\hline Female & 28 & 19 & & 25 & 22 & \\
\hline Age & & & 0.284 & & & 0.872 \\
\hline$\leq 60$ & 15 & 19 & & 17 & 17 & \\
\hline$>60$ & 51 & 42 & & 48 & 45 & \\
\hline Alcohol abuse & & & 0.372 & & & 0.698 \\
\hline Yes & 22 & 25 & & 23 & 24 & \\
\hline No & 44 & 36 & & 42 & 38 & \\
\hline Smoking & & & 0.803 & & & 0.665 \\
\hline Yes & 31 & 30 & & 30 & 31 & \\
\hline No & 35 & 31 & & 35 & 31 & \\
\hline Location & & & 0.971 & & & 0.632 \\
\hline Upper & 29 & 27 & & 30 & 26 & \\
\hline Middle/lower & 37 & 34 & & 35 & 36 & \\
\hline Length, cm & & & 0.035 & & & 0.623 \\
\hline$\leq 4$ & 35 & 21 & & 27 & 29 & \\
\hline$>4$ & 31 & 40 & & 38 & 33 & \\
\hline Tstage & & & 0.790 & & & 0.728 \\
\hline $\mathrm{T} 1-2$ & 12 & 10 & & 12 & 10 & \\
\hline T3-4 & 54 & 51 & & 53 & 52 & \\
\hline Lymph node metastasis & & & 0.037 & & & 0.014 \\
\hline NO-1 & 59 & 46 & & 59 & 46 & \\
\hline N2-3 & 7 & 15 & & 6 & 16 & \\
\hline Metastasis & & & 0.057 & & & 0.333 \\
\hline MO & 56 & 58 & & 60 & 54 & \\
\hline M1 & 10 & 3 & & 5 & 8 & \\
\hline TNM stage & & & 0.015 & & & 0.378 \\
\hline$|-| \mid$ & 22 & 9 & & 18 & 13 & \\
\hline III-IV & 44 & 52 & & 47 & 49 & \\
\hline
\end{tabular}

region ( $p=0.017$, Fig. 3d). Moreover, ChIP-sequencing analysis on two highly correlated biological replicates of KYSE 150 identified the recruitment of NRF2 to the SLC7A11 promoter. Strikingly, a strong enrichment of NRF2 signals was detected within the promoter regions of SLC7A11 which is located at chromosome 4, indicating that there is an interaction between NRF2 and SLC7A11 (Fig. 3e).

\section{Relationship between SLC7A11 or NRF2 nuclear expression} and clinical response CRT

Eleven patients (8.7\%) achieved a complete response, 67 patients achieved a partial response, 30 patients (23.6\%) experienced stable disease, and 19 patients experienced progressive disease (Fig. 4a, b.). The treatment response was poorer in patients with high NRF2 nuclear expression than in patients with low NRF2 nuclear expression ( $47.5 \%$ vs $74.2 \%, p=0.002)$. Similarly, patients with high expression of SLC7A11 had a poorer treatment response than patients with low SLC7A11 expression (51.6\% vs $70.8 \%, p=0.027$; Table 4 ).

\section{Effect of the NRF 2 and SLC7A11 expression on overall} survival (OS) and progression-free survival (PFS) in ESCC

The NRF2-positive patients had a shorter OS (20 vs 32 months, $p=0.006$; Fig. 5a) and PFS (12 vs 20 months, $p=0.021$; Fig. 5 d) than the NRF2-negative group. Similarly, SC7A11-positive patients had a shorter OS (19.5 vs 34 months, $p=0.002$; Fig. $5 \mathrm{~b}$ ) and PFS (13 vs 22 months, $p=0.007$; Fig. 5e) than SLC7A11-negative patients. Patients negative for both NRF2 and SLC7A11 had the longest OS (mean OS; 30.5 months), whereas double positive expression was linked to the shortest OS (mean 


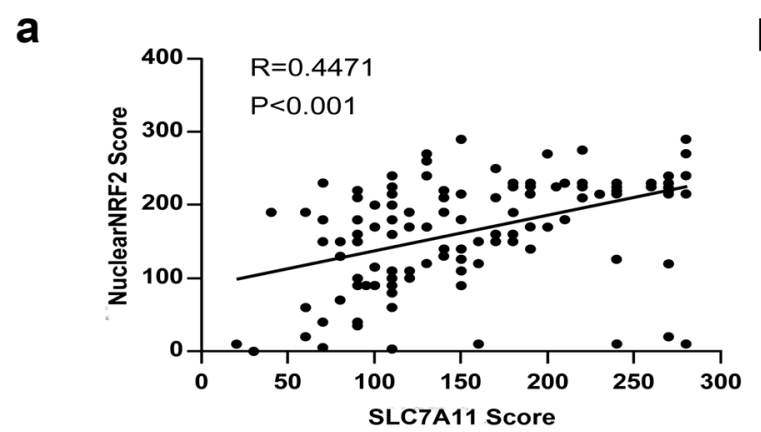

C >SLC7A11 promoter ATTTTGGTTTTATAAAAACTTTAA CTTGGTGTAGCTGAAACCAATTA TATAGAATTTAGATAGATAATTTA AAAAGGAGAAAATGTAGCCTCAA TTATTTGGATGGCAAAGCATTGA NRF2 GGTGGTGTCATTTTC b

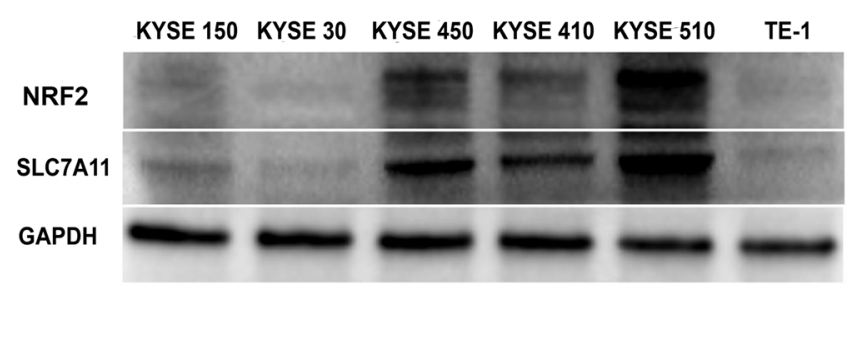

e

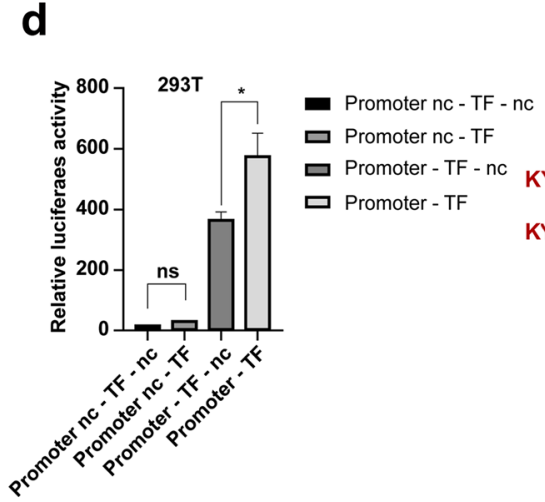

Fig. 3 Correlation between SLC7A11 and NRF2 expression. a SLC7A1 1 expression was positively correlated with nuclear NRF2 expression among all the patients $(n=127)$. b SLC7A11 and NRF2 protein expression were analyzed in six ESCC cell lines. Western blotting revealed that SLC7A11 expression positively correlated with NRF2 level. c Sequence of the SLC7A11 promoter showing the position of the most representative putative NRF2 binding sites of the promoter. Schematic of putative NRF2 binding sites in the proximal region of SLC7A11 promoter. $\mathbf{d}$ Dual-luciferase reporter assays showing NRF2 acting as a trans-activator of SLC7A1 1 promoter activity. Luciferase activity assays $(n=3)$ were performed after transfection of either firefly luciferase promoter plasmid, NRF2 overexpression plasmid (TF), or TF-nc plasmid into 293T cells. Data are presented as the mean \pm SEM. ${ }^{*} p<0.05$. e Integrative Genomics Viewer (IGV) snapshots of NRF2 binding sites at the promoter of SLC7A11 region
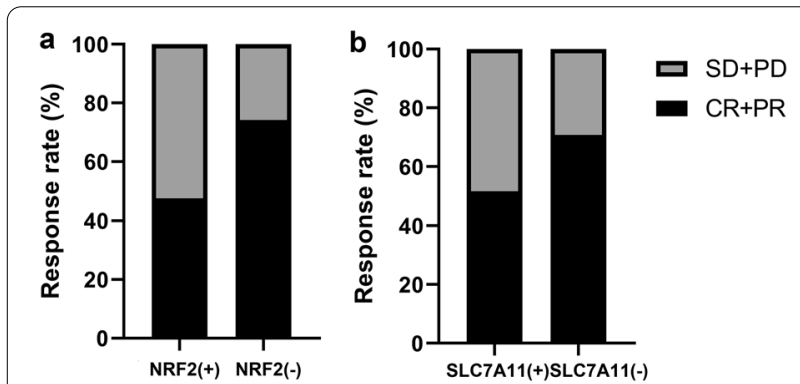

Fig. 4 Relationship between SLC7A11 or NRF2 nuclear expression and clinical response to CRT. SLC7A11 (a) and nuclear NRF2 (b) treatment responses of ESCC patients. CR: complete response; PR: partial response; SD: stable disease; PD: progressive disease

OS: 12 months); patients positive for either nuclear expression of NRF2 or SLC7A11 had a similar OS (13 vs 15 months, $p=0.012$; Fig. $5 \mathrm{c}$ ). Additionally, patients positive for both NRF2 and SLC7A11 had a shorter mean PFS than those negative for both (17 vs 58 months,
Table 4 Relationship between SLC7A11 or nuclear NRF2 expression and clinical response to CRT

\begin{tabular}{lllll}
\hline & & \multicolumn{2}{c}{ Treatment response } & \\
\cline { 3 - 4 } & & CR + PR & SD + PD & P-value \\
\hline \multirow{2}{*}{ Nuclear NRF2 } & Low expression (\%) & $49(74.2)$ & $17(25.8)$ & 0.002 \\
& High expression (\%) & $29(47.5)$ & $32(52.5)$ & \\
\multirow{3}{*}{ LLC7A11 } & Low expression (\%) & $46(70.8)$ & $19(29.2)$ & 0.027 \\
& High expression (\%) & $32(51.6)$ & $30(48.4)$ & \\
\hline
\end{tabular}

$p=0.003$; Fig. 5f), while patients that were positive for either nuclear expression of NRF2 or SLC7A11 had similar PFS (29 vs 26 months, $p=0.741$; Fig. 5f). These findings suggest that analyzing NRF2 nuclear expression and SLC7A11 expression status may predict ESCC prognosis received CRT. 

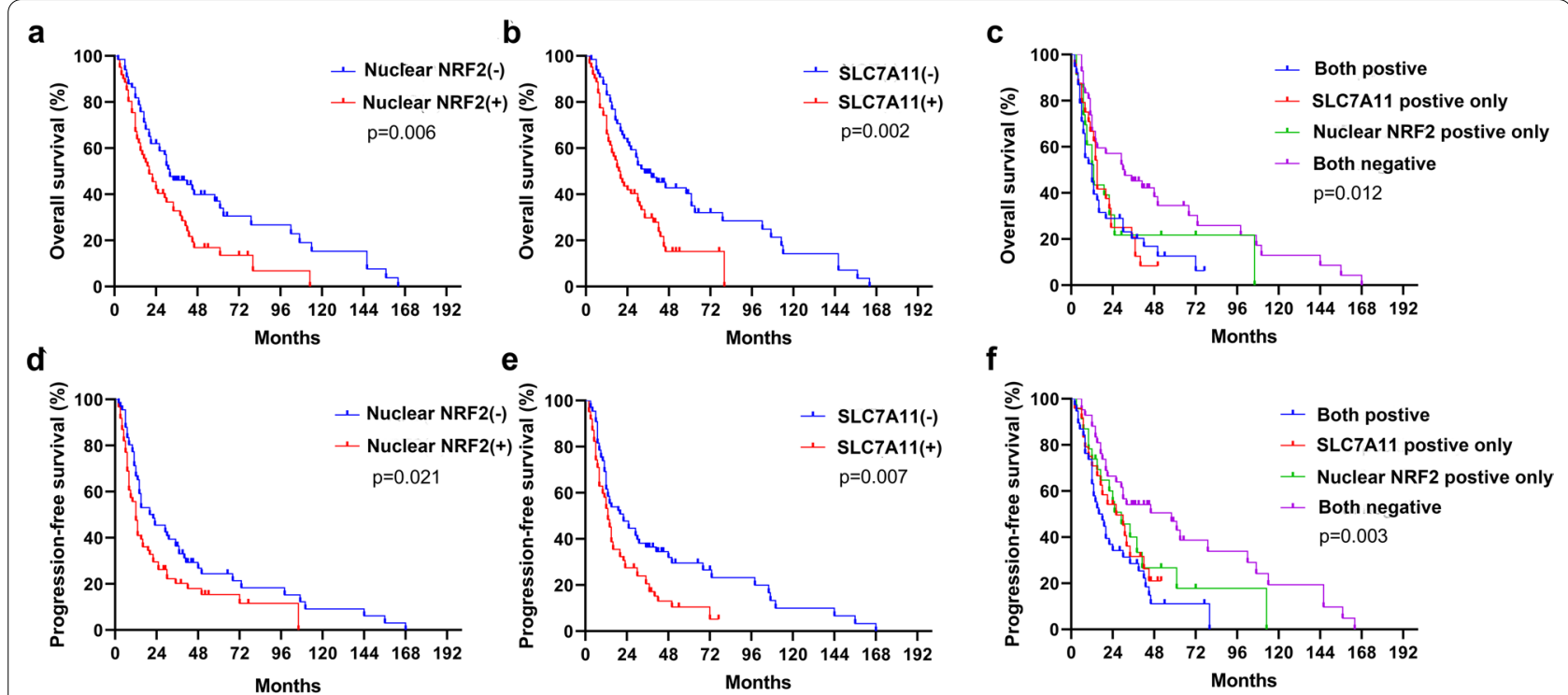

Fig. 5 Kaplan-Meier survival curves for NRF2 nuclear expression and SLC7A11 expression. OS survival curves for nuclear NRF2 (a), SLC7A11 (b), and their combined (c) expression. PFS survival curves for nuclear NRF2 (d), SLC7A11 (e), and their combined (f) expression

\section{Prognostic value in ESCC}

Next, univariate and multivariate Cox regression analysis were used to assess possible prognostic factors in patients with ESCC (Table 5). Univariate analysis revealed that NRF2 nuclear expression and SLC7A11 expression, sex, tumor location, tumor length, $\mathrm{T}$ stage, lymph node and distant metastasis, and TNM stage were all associated with OS; conversely, age, alcohol and smoking history were not. Subsequent multivariate Cox regression analysis revealed that sex, tumor location, and NRF2 nuclear expression and SLC7A11 expression were independent prognostic factors for OS.

\section{SLC7A11 overexpression induced by hyperactive NRF2 promotes radioresistance by inhibiting ferroptosis}

We found a strong correlation between nuclear expression of NRF2 (indicating NRF2 activation) and SLC7A11 expression, relating to poor CRT response and shorter OS and PFS in ESCC patients. We reasoned that hyperactive NRF2 promotes radioresistance partly through SLC7A11-mediated ferroptosis inhibition in ESCC. Therefore, we examined whether stabilized NRF2 expression would promote radioresistance by inhibiting ferroptosis in ESCC cell lines. We constructed NRF2 overexpression (NF-OE) in KYSE 30 and KYSE 150 cell lines (Fig. 6a). Considering that NRF2 is an important regulator of the cellular ROS levels $[28,29]$ and the accumulation of ROS is the trigger for ferroptosis [30, 31], we validated cellular ROS levels and confirmed that (via DCFDA staining, details in Methods and Materials) NRF2 overexpression alleviated the total ROS levels induced by RT in ESCC cells (Additional file 1: Figure S1a). In addition, we found that NRF2 overexpression significantly reduced RT-induced lipid peroxidation levels (analyzed by C11-BODIPY staining), as well as ferroptosis marker gene (PTGS2) expression (Fig. 6b, c) [32]. TEM revealed that RT-treated KYSE 150 (Fig. 6d) and KYSE 30 (Additional file 1: Figure S1b) cells exhibited shrunken mitochondria, increased membrane density and ruptured outer mitochondrial membranes [30]. These are significant ferroptosis-related morphological features [10], including autophagy, which under the exposure of radiation, has been proved to be associated with ferroptosis [33] (such as autophagosomes; Fig. 6d). These phenomena were all largely improved in NRF2 overexpression cells after RT treatment. Consistent with prior literature [34, 35], the data indicated that NRF2 overexpression could promote radioresistance (Fig. 6e-g; Additional file 1: Figure S1c, d).

Notably, the effect of NRF2 overexpression on radioresistance was abrogated under ferroptosis inhibitor ferrostatin-1 treatment (Fig. 6e-g; Additional file 1: Figure S1c, d), suggesting that NRF2 nuclear expression may promote radioresistance by inhibiting ferroptosis; additionally, we found that NRF2 overexpression substantially increased SLC7A11 expression in ESCC cell lines (Fig. 7a). We then knocked down SLC7A11 with siRNA in NRF2 overexpression cells (NF-OE-SLC-Si) to test our hypothesis (Fig. 7b). SLC7A11 deletion in NF-OE KYSE 150 cell lines restored the cellular total ROS levels (Additional file 2: Figure S2a), lipid peroxidation levels and PTGS2 expression reduction induced by RT (Fig. 7c, d), 
Table 5 Univariate and multivariate analyses of prognostic markers for overall survival in ESCC

\begin{tabular}{|c|c|c|c|c|c|c|}
\hline \multirow[t]{2}{*}{ Variable } & \multicolumn{3}{|c|}{ Univariate analysis } & \multicolumn{3}{|c|}{ Multivariate analysis } \\
\hline & HR & $95 \% \mathrm{Cl}$ & P-value & $\mathrm{HR}$ & $95 \% \mathrm{Cl}$ & P-value \\
\hline Nuclear NRF2 expression & & & 0.005 & & & 0.002 \\
\hline High vs. low or no & 1.802 & $1.191-2.729$ & & 1.908 & $1.256-2.899$ & \\
\hline SLC7A11 expression & & & 0.004 & & & 0.010 \\
\hline Positive vs. negative & 1.858 & $1.220-2.829$ & & 1.767 & $1.146-2.725$ & \\
\hline Sex & & & 0.019 & & & 0.022 \\
\hline Male vs. Female & 0.598 & $0.389-0.919$ & & 0.6 & $0.389-0.927$ & \\
\hline Age (years) & & & 0.067 & & & \\
\hline$\leq 60$ vs. $>60$ & 1.583 & $0.969-2.584$ & & & & \\
\hline Alcohol abuse & & & 0.200 & & & \\
\hline Yes vs. No & 1.318 & $0.864-2.011$ & & & & \\
\hline Smoking & & & 0.444 & & & \\
\hline Yes vs. No & 1.172 & $0.781-1.758$ & & & & \\
\hline Location & & & 0.002 & & & 0.002 \\
\hline Upper vs. Middle/Lower & 1.898 & $1.268-2.839$ & & 1.908 & $1.256-2.899$ & \\
\hline Length $(\mathrm{cm})$ & & & 0.038 & & & \\
\hline$\leq 4$ Vs. $>4$ & 1.566 & $1.025-2.394$ & & & & \\
\hline Tstage & & & 0.007 & & & \\
\hline T1-2 vs. T3-4 & 1.542 & $1.126-2.112$ & & & & \\
\hline Lymph node metastasis & & & 0.001 & & & \\
\hline N0-1 vs. N2-3 & 1.741 & $1.243-2.437$ & & & & \\
\hline Distant metastasis & & & 0.004 & & & \\
\hline M0 vs. M1 & 2.404 & $1.321-4.375$ & & & & \\
\hline TNM stage & & & 0.001 & & & \\
\hline I-II vs. III-IV & 1.634 & $1.220-2.188$ & & & & \\
\hline Treatment strategy & & & 0.606 & & & \\
\hline CRT vs. RT & 0.888 & $0.566-1.394$ & & & & \\
\hline
\end{tabular}

HR: Hazard ratio; Cl: confidence interval

${ }^{*} p<0.05$

and RT sensitivity was regained (Fig. 7e-g; Additional file 2: Figure S2b, c). Similarly, the effect of SLC7A11 deficiency was abolished with ferrostatin-1 treatment with RT (Fig. 7e-g; Additional file 2: Figure S2b, c). These results suggest that NRF2 overexpression in ESCC cells partly promotes radioresistance through SLC7A11-mediated ferroptosis inhibition.

\section{Discussion}

Numerous studies have recently reported that the hyperactivation of NRF2 contributes to radioresistance in ESCC $[14,16,36]$; however, the underlying mechanism of NRF2-mediated radioresistance remains unclear. Furthermore, accumulating evidence has demonstrated that SLC7A11 promotes therapy resistance partly by suppressing ferroptosis, a form of programmed cell death, that is, induced by excessive lipid peroxidation $[10,12,37,38]$. In this study, we examined the expression of SLC7A11 and NRF2 in patients with ESCC who received CRT and evaluated their association with clinicopathological characteristics, long-term prognosis, and therapeutic efficacy. Interestingly, the results of clinical analyses revealed a positive correlation between the expression of NRF2 and SLC7A11, indicating a worsening of therapy response and poor prognosis. Emerging evidence demonstrates that the NRF2 signaling pathway plays a significant role in mediating ferroptosis [39-41] and the coeffect between NRF2 and SLC7A11 [42]. We propose that NRF2-associated radioresistance is largely induced by the SLC7A11-mediated inhibition of ferroptosis. To validate this notion, we performed further in vitro validation studies, which are crucial for highlighting the potential of the NRF2/SLC7A11/ferroptosis axis as a possible therapeutic target against biomarkers of therapy resistance.

The nuclear transcription factor, NRF2, is an essential regulator of antioxidant response elements, and the activation of NRF2 signaling plays an important role in 


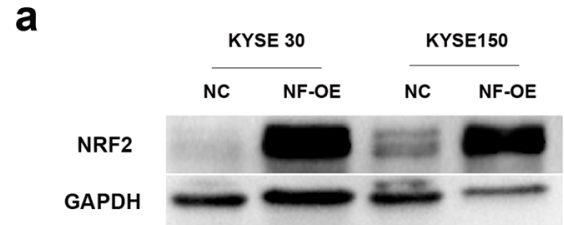

C

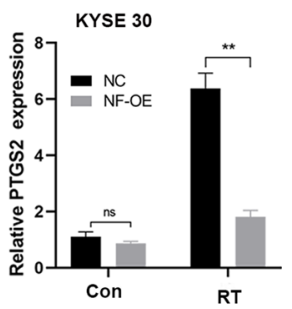

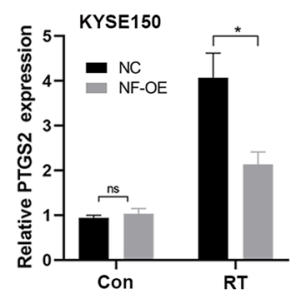

b
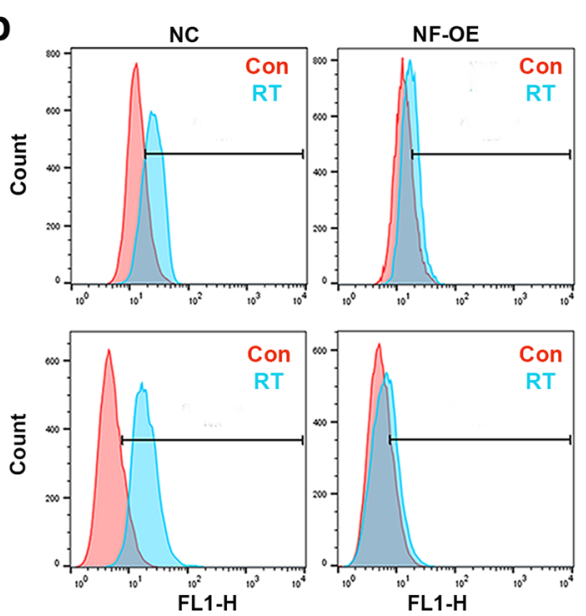
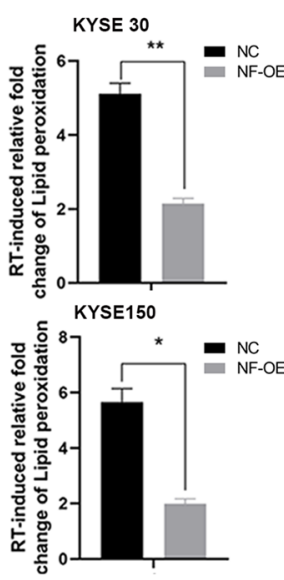

d

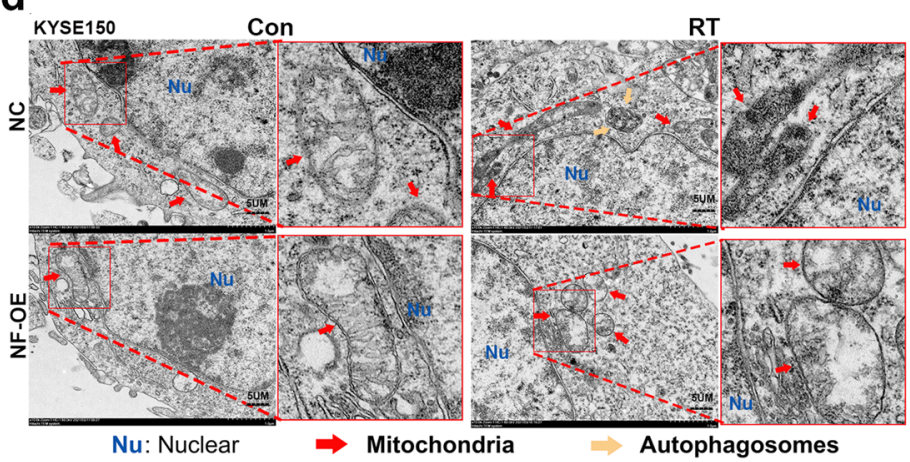

e
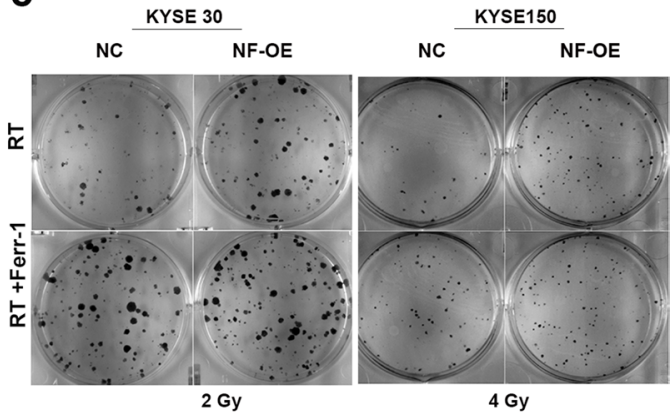

f
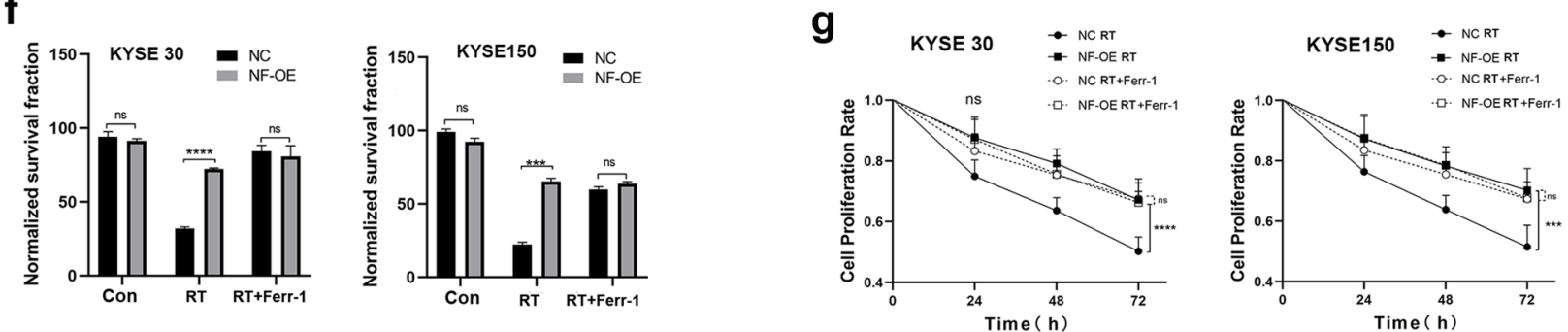

Fig. 6 Hyperactive NRF2 promotes radioresistance by inhibiting ferroptosis in ESCC cells. a Western blot analysis showing NRF2 levels in KYSE 30 and KYSE 150 cell lines with overexpression of NRF2 (NF-OE), and negative control (NC). $\mathbf{b}$ Lipid peroxidation assessment of NC and NF-OE $24 \mathrm{~h}$ after 6 Gy RT in KYSE 30 and KYSE 150 cells, respectively. Bar graph showing RT-induced relative fold changes of lipid peroxidation levels per 10,000 cells by C11-BODIPY staining in the indicated cells. c Quantitative RT-PCR analysis of PTGS2 expression in NC and NF-OE KYSE 30 or KYSE 150 cells $24 \mathrm{~h}$ after 6 Gy RT, respectively. d TEM images of NC and NF-OE KYSE 150 cells $24 \mathrm{~h}$ after exposure to 6 Gy of RT. Nu: nucleus; red arrows: mitochondria; yellow arrows: autophagosome; Scale bars: 5 m. e Representative images of clonogenic survival assays in NC and NF-OE KYSE 30 or KYSE 150 cells pre-treated with $5 \mu \mathrm{M}$ ferrostatin-1 or DMSO for $24 \mathrm{~h}$, followed by exposure to $2 \mathrm{~Gy}$ or $4 \mathrm{~Gy}$ of RT, respectively. f The quantified clonogenic survival assay in NC and NF-OE KYSE 30 or KYSE 150 cells that were pre-treated with $5 \mu \mathrm{M}$ ferrostatin-1 or DMSO for $24 \mathrm{~h}$ followed by 2 Gy or 4 Gy of RT. g Quantified cell proliferation assay curves analyzed by CCK-8 in NC and NF-OE KYSE 30 or KYSE 150 cells pre-treated with $5 \mu$ M ferrostatin-1 or DMSO for $24 \mathrm{~h}$, followed by exposure to $6 \mathrm{~Gy}$ RT. Each experiment was conducted independently triplicate. Student's t-test (two-tailed) was used for statistical analysis, ${ }^{*} p<0.05,{ }^{* *} p<0.01,{ }^{* * *} p<0.001,{ }^{* * * *} p<0.0001$. Bar graphs: mean \pm SEM, $n=3$

protecting cells and tissues from oxidative stress-induced injury [43]. Increasing evidences have demonstrated that NRF2 promotes radioresistance in ESCC [16, 36, 44]; however, the precise mechanistic evidence remains to be elucidated. It has been additionally reported that NRF2 functions as a driver gene for promoting the progression of cancer and metastasis in numerous tumor types, including lung cancer, breast carcinoma cells, and ESCC 


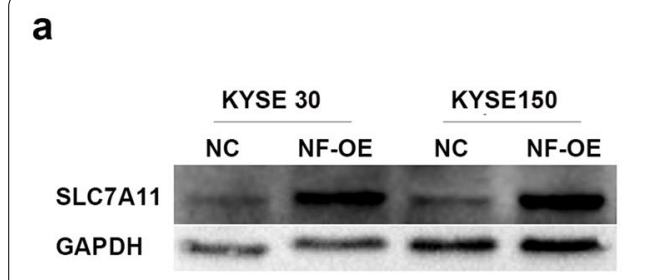

C
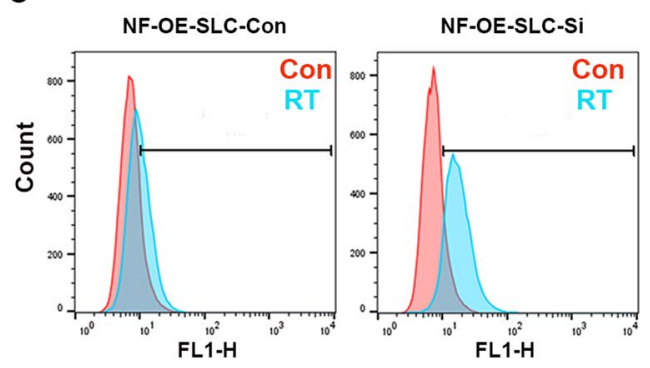

e

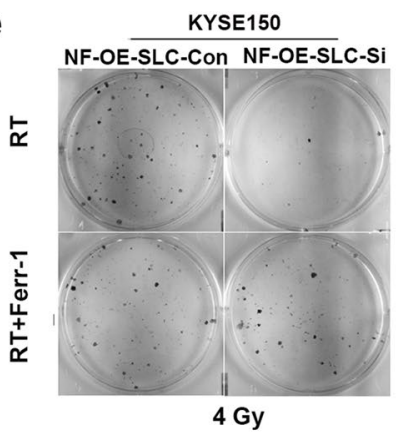

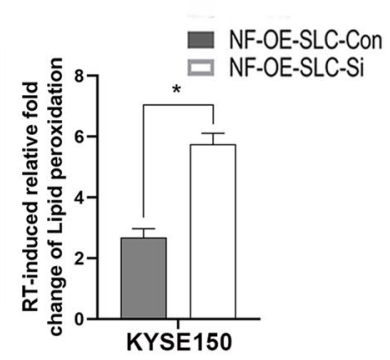

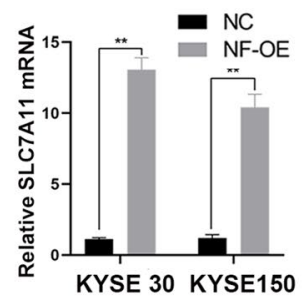

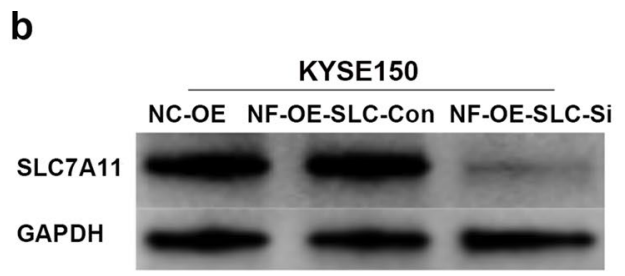

d

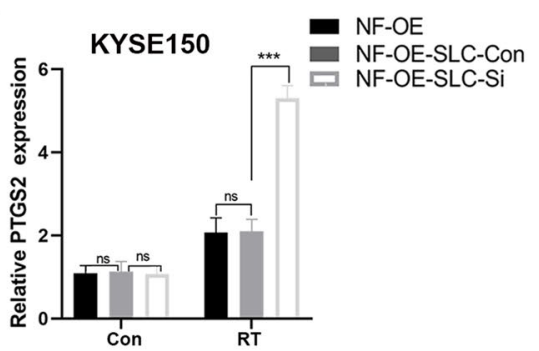

g

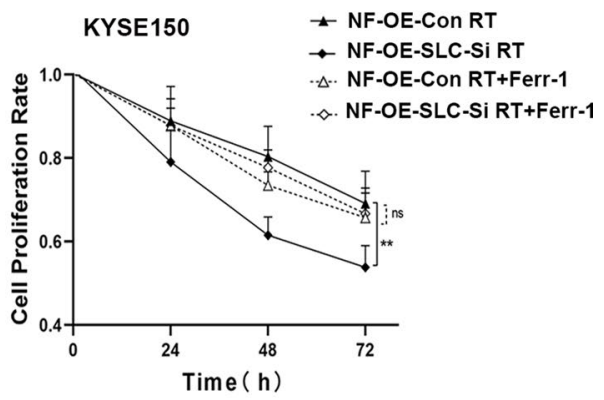

Fig. 7 Hyperactive NRF2 inhibits RT-induced ferroptosis by regulating SLC7A11 in ESCC cells. a Western blotting and qRT-PCR analysis of NRF2 and SLC7A11 expression in NC and NF-OE KYSE 30 and KYSE 150 cells. b Western blot analysis of SLC7A11 levels in NF-OE KYSE 150 cells with SLC7A11 siRNA (NF-OE-SLC-Si), negative control (NF-OE-SLC-Con), and NF-OE KYSE 150. $\mathbf{c}$ Lipid peroxidation assessment in NF-OE-SLC-Si and NF-OE-SLC-Con KYSE 150 cells $24 \mathrm{~h}$ after exposure to $6 \mathrm{~Gy}$ of RT. Bar graph showing RT-induced relative fold changes of lipid peroxidation levels per 10,000 cells by C11-BODIPY staining in the indicated cells. $\mathbf{d}$ qRT-PCR analysis of PTGS2 expression in NF-OE-SLC-Si and NF-OE-SLC-con KYSE 150 cells $24 \mathrm{~h}$ after exposure to 6 Gy of RT. e Representative images of clonogenic survival assays in NF-OE-Con and NF-OE-SLC-Si KYSE 150 cells pre-treated with $5 \mu \mathrm{M}$ ferrostatin-1 or DMSO for $24 \mathrm{~h}$, followed by exposure to $4 \mathrm{~Gy}$ of RT, respectively. f The quantified clonogenic survival assay in NF-OE-Con and NF-OE-SLC-Si KYSE 150 cells that were pre-treated with $5 \mu$ M ferrostatin-1 or DMSO for $24 \mathrm{~h}$ followed by 4 Gy of RT, respectively. $\mathbf{g}$ Quantified cell proliferation assay curves analyzed by CCK-8 in NF-OE-SLC-con and NF-OE-SLC-Si KYSE 150 cells pre-treated with $5 \mu$ M ferrostatin-1 or DMSO for $24 \mathrm{~h}$, followed by exposure to 6 Gy RT. Each experiment was conducted independently triplicate. Student's t-test (two-tailed) was used for statistical analysis, ${ }^{*} p<0.05,{ }^{* *} p<0.01,{ }^{* * *} p<0.001,{ }^{* * * *} p<0.0001$. Bar graphs: mean \pm SEM, $\mathrm{n}=3$

$[13,45,46]$. However, Li et al. suggested that NRF2 could act as a tumor suppressor in ESCC [2]. Here, the results of our clinical analyses revealed that the overexpression of NRF2 was associated with poor therapeutic outcomes and prognosis in patients with ESCC who received CRT. Moreover, several studies have recently demonstrated that the relevance of the NRF2-lipid peroxidation-ferroptosis axis [40, 41, 47], while another study has reported that the activation of NRF2 signaling alleviates ferroptosis [19].

Ferroptosis, a form of regulated cell death that is different from other forms of cell death, such as apoptosis and necrosis [48], is induced by excessive lipid peroxidation, and it serves as a natural mechanism for tumor inhibition. Targeting ferroptosis represents a promising strategy for cancer treatment, as it helps overcome tumor resistance [29]. The growing recognition of NRF2 as a modulator of ferroptosis indicates that it regulates the sensitivity of cancer treatment in specific types of cancer $[49,50]$. For instance, the suppression of NRF2 expression reverses GPX4 inhibitor-induced ferroptosis resistance in head and neck tumors [51] and artesunateinduced ferroptosis resistance in cisplatin-resistant head and neck cancer cells [52]. NRF2 is a major factor that 
regulates the effects of ferroptosis-related therapies on hepatocellular carcinoma [39]. Erastin, an inducer of ferroptosis, and acetaminophen promote the death of nonsmall cell lung cancer cells by regulating NRF2 expression [53]. However, their relevance in ESCC radioresistance remains unexplored. Our in vitro data revealed that the overexpression of NRF2 promoted radioresistance in ESCC, largely by inhibiting RT-induced ferroptosis, and the effect of NRF2 overexpression on radioresistance was abrogated by treatment with the ferroptosis inhibitor ferrostatin-1. In recent years, several studies have indicated that ROS-mediated therapeutic resistance is closely related to the regulation of NRF2 expression [54], and ROS-induced lipid peroxidation plays an essential role in ferroptosis [31, 55]. Mechanistically, in the present study, we revealed that the activation of NRF2 weakened radiation-induced ROS generation and lipid peroxidation, alleviating RT-induced ferroptosis and promoting radioresistance in ESCC. We additionally proposed that activated NRF2 could directly combine with the promoter region of SLC7A11 to regulate its transcription, which is related to the inhibition of ferroptosis $[56,57]$.

SLC7A11, an important component of the cystine/ glutamate transporter, regulates cellular lipid peroxidation, and suppresses ferroptosis [58]. Recent studies have demonstrated that SLC7A11 plays diverse functional roles, including the regulation of therapeutic resistance in cancer [38]. Numerous studies have confirmed that therapeutic resistance is associated with SLC7A11 [38]. For instance, the study by Lei et al. demonstrated that the overexpression of SLC7A11 promotes radioresistance, largely via the inhibition of ferroptosis [10]; It has been additionally demonstrated that SLC7A11 is related to radioresistance in human breast cancer and murine melanoma cell lines $[59,60]$. Cobler et al. reported that SLC7A11-specific therapeutics would provide tumorspecific radiosensitivity to $\gamma$ radiation in breast cancer cells, which allows the use of lower radiation doses in treatments, thus producing fewer side effects than other proposed sensitizers [59]. Additionally, previous studies have reported that SLC7A11 may be a potential target gene of NRF2 [42]. For example, Fan et al. [19] mentioned that the activation of NRF2-Keap1 signaling upregulates SLC7A11 and amplifies the secretion of glutamate, thereby affecting the tumor microenvironment [19], while another study demonstrated that the retinal expression of SLC7A11 is regulated by NRF2 in patients with diabetes [61]. The depletion of glutathione in the cortex is associated with the activation of the NRF2 and nuclear factor kappa $\mathrm{B}$ signaling pathways, leading to the upregulation of SLC7A11 expression [62]. However, Ono et al. reported that NRF2 does not contribute to the upregulation of SLC7A11 expression through polyunsaturated fatty acids [63]. The results of our IHC analyses revealed that the expression levels of SLC7A11 were consistent with those of NRF2, indicating that there was a significant positive correlation between the expression of SLC7A11 and NRF2. We also observed that the expression of NRF2 substantially increased the expression of SLC7A11 in ESCC cell lines. Interestingly, the results of our dual-luciferase reporter assay and ChIP-sequencing analysis revealed that NRF2 directly binds to the promoter region of SLC7A11 to regulate the expression of SLC7A11. These results support the interaction between NRF2 and SLC7A11 in ESCC and describe their underlying influence on treatment response and prognosis.

As mentioned above, despite the accumulating evidence on the relationship between NRF2 and SLC7A11 expression, in addition to their effect on radioresistance in various types of cancers, the effect of SLC7A11 and NRF2 on radiosensitivity in ESCC remains to be elucidated. We analyzed the correlation between the clinicopathological characteristics, including the expression of SLC7A11 and NRF2 and the efficacy of CRT, and the results demonstrated that higher expression levels of SLC7A11 and NRF2 were related to a poor therapy response in patients with ESCC who received CRT. We additionally investigated whether the inhibition of SLC7A11 expression sensitizes NRF2-overexpressing ESCC tumor cells to RT by inducing ferroptosis. We observed that pre-treatment with SLC7A11-siRNA potently sensitized NRF2 overexpression in vitro. Altogether, our results demonstrate that NRF2 partly mediates RT resistance via the SLC7A11-mediated inhibition of ferroptosis.

Additionally, growing evidence has demonstrated that SLC7A11 is overexpressed in various types of cancers and is associated with patients' poor prognosis [38]. Shiozaki et al. suggested that the expression of SLC7A11 could affect the G1/S checkpoint in ESCC cells as well as the prognosis of patients with ESCC who have undergone esophagectomy [7]. However, the correlation between the expression of SLC7A11 and prognosis of patients with ESCC who received CRT has not been investigated to date. Zhang et al. have previously reported the prognostic value of SLC7A11 in patients with liver cancer [64]. The study by Ma et al. demonstrated that the overexpression of SLC7A11 is a prognostic factor for poor rates of OS and high rates of postoperative recurrence in laryngeal squamous cell carcinoma [21]. In the current study, univariate and multivariate analysis results revealed that the expression of SLC7A11 is an independent prognostic factor for ESCC. We found that high levels of NRF2 or SLC7A11 were associated with low OS, PFS, and poor treatment response in patients with ESCC. Zhong et al. reported that the expression of SLC7A11 
promotes metastasis, which is related to a reduced survival rate in patients with prostate cancer [65]. Similarly, we observed that the expression of SLC7A11 was positively correlated with lymph node metastasis, suggesting that SLC7A11 may have a prognostic effect on patients with ESCC via the regulation of lymph node invasion. This indicated that the expression of SLC7A11 might be a potentially useful indicator for selecting irradiated areas of lymphatic drainage. Therefore, SLC7A11 may serve as a potential novel target for cancer therapy and may enhance radiosensitivity.

Taken together, our results provide definitive evidence linking NRF2 with SLC7A11-mediated ferroptosis and elucidate the radioresistance of ESCC. We speculate that a better understanding of the underlying tumor contexts would maximize the efficacy of cancer therapy. NRF2/ SLC7A11/ferroptosis-specific therapeutics may provide tumor-specific sensitization to RT in patients with ESCC who develop de novo radioresistance. The exploration of the combination of NRF2/SLC7A11 inhibitors or ferroptosis inducers and radiotherapy remains an important area for future research.

\section{Conclusions}

In summary, the results of this study demonstrated that SLC7A11 plays an important role in the radiosensitivity of ESCC cells by interacting with NRF2, and that expression of SLC7A11 is related to the radiosensitivity and long-term prognosis of ESCC following RT. To the best of our knowledge, the present study is the first to examine the correlation between SLC7A11 and NRF2 and investigate the clinicopathological and prognostic significance of SLC7A11 expression through IHC analysis of ESCC samples from patients who received RT. Immunohistochemical staining showed that the expression of SLC7A11 was an independent prognostic factor in patients with ESCC who received CRT. Furthermore, the study revealed that ESCC radiosensitivity could be potentially improved by targeting the NRF2/SLC7A11/ ferroptosis pathway. An greater understanding of the role of SLC7A11 might lead to its use as an important biomarker in ESCC.

\footnotetext{
Abbreviations

SLC7A11: Solute carrier family 7 member 11; NRF2: Nuclear factor erythroid-2; ChIP: Chromatin immunoprecipitation; ESCC: Esophageal squamous cell carcinoma; CRT: Chemoradiotherapy; CT: Chemotherapy; IHC: Immunohistochemistry; OS: Overall survival; PBS: Phosphate buffered solution; PFS: Progressionfree survival; PR: Partial response; CR: Complete response; SD: Stable disease; PD: Progressive disease; OD: Optical density; ROS: Reactive oxygen species; RT: Radiotherapy; TEM:Transmission electron microscopy.
}

\section{Supplementary Information}

The online version contains supplementary material available at https://doi. org/10.1186/s12967-021-03042-7.

Additional file 1: Figure S1. Hyperactive NRF2 promotes radioresistance by inhibiting ferroptosis in ESCC cells. (a) Total ROS levels assessment in NC and NF-OE KYSE 30 and KYSE 150 cells at $24 \mathrm{~h}$ after exposure to $6 \mathrm{~Gy}$ of RT. Bar graph showing RT-induced relative fold changes of cellular total ROS levels per 10000 cells via DCFDA staining in the indicated cells. (b) TEM images of NC and NF-OE KYSE 30 cells $24 \mathrm{~h}$ after exposure to 6 Gy of RT. Nu, nucleus; red arrows, mitochondria. Scale bars: $5 \mu \mathrm{m}$. (c) Representative images of clonogenic survival assays in NC and NF-OE KYSE 30 or KYSE 150 cells pre-treated with $5 \mu \mathrm{M}$ ferrostatin-1 or DMSO for $24 \mathrm{~h}$, followed by exposure to 0 Gy, 2 Gy, 4 Gy or 6 Gy of RT, respectively. (d) The quantified clonogenic survival assay in NC and NF-OE KYSE 30 or KYSE 150 cells that were pre-treated with $5 \mu \mathrm{M}$ ferrostatin-1 or DMSO for $24 \mathrm{~h}$ followed by 0 Gy, 2 Gy, 4 Gy or 6 Gy of RT, respectively. Each experiment was conducted independently triplicate. Student's t-test (two-tailed) was used for statistical analysis, ${ }^{*} p<0.05,{ }^{* *} p<0.01,{ }^{* * *} p<0.001,{ }^{* * * *} p<0.0001$. Bar graphs: mean $\pm S E M, n=3$.

Additional file 2: Figure S2. Hyperactive NRF2 inhibits RT-induced ferroptosis by regulating SLC7A11 in ESCC cells. (a) Total ROS levels assessment in NF-OE-SLC-Con and NF-OE-SLC-Si KYSE 150 cells at $24 \mathrm{~h}$ after exposure to 6 Gy of RT. Bar graph showing RT-induced relative fold changes of cellular total ROS levels per 10000 cells by DCFDA staining in the indicated cells. (b) Representative images of clonogenic survival assays in NF-OE-SLC-Con and NF-OE-SLC-Si KYSE 150 cells pre-treated with $5 \mu \mathrm{M}$ ferrostatin-1 or DMSO for 24 h, followed by exposure to 0 Gy, 2 Gy, or 6 Gy of RT, respectively. (c) The quantified clonogenic survival assay in NF-OESLC-Con and NF-OE-SLC-Si KYSE 150 cells that were pre-treated with $5 \mu \mathrm{M}$ ferrostatin-1 or DMSO for $24 \mathrm{~h}$ followed by $0 \mathrm{~Gy}, 2$ Gy or 6 Gy of RT, respectively. Each experiment was conducted independently triplicate. Student's t-test (two-tailed) was used for statistical analysis, ${ }^{*} p<0.05,{ }^{* *} p<0.01$, ${ }^{* * *} p<0.001,{ }^{* * * *} p<0.0001$. Bar graphs: mean \pm SEM, $n=3$.

\section{Acknowledgements}

We would like to thank Editage (www.editage.cn) for English language editing

\section{Authors' contributions}

LF: conceptualization, data collection, statistics, original draft; KZ: data collection, formal analysis, resources; LS, XY, JZ, CL: conceptualization, review, and editing of the manuscript; BL: obtained funding and supervised study. All authors provided final approval of the submitted and published versions. All authors read and approved the final manuscript.

\section{Funding}

This work was supported by National Natural Science Foundation of China (No. 81874224), Academic promotion program of Shandong First Medical University (2019LJ004), National Key Research and Development Program of China (2016YFC0105106), Taishan Scholar Construction Project (ts20120505), Tumor prevention and control Joint Fund of Shandong province natural science fund (ZR2019LZL008), Yantai Science and Technology Innovation Development Program (2020YT06121070).

Availability of data and materials

Data are available from the authors upon reasonable request.

\section{Declarations}

Ethics approval and consent to participate

This study was approved by the Ethics Committee of Shandong Cancer Hospital and Institute (approval number: SDTHEC201803); the requirement of informed consent was waived due to the retrospective nature of the study.

Consent for publication

Not applicable. 


\section{Competing interests}

The authors declare that the research was conducted in the absence of any commercial or financial relationships that could be construed as a potential conflict of interest.

\section{Author details}

${ }^{1}$ Shandong First Medical University and Shandong Academy of Medical Sciences, Jinan 250062, Shandong, China. ' Department of Radiation Oncology, Shandong Cancer Hospital and Institute, Shandong First Medical University, Academy of Medical Sciences, Jinan 250117, China. ${ }^{3}$ Department of Radiation Oncology, Yantai Affiliated Hospital of Binzhou Medical University, Yantai 264100, Shandong, China.

\section{Received: 21 May 2021 Accepted: 14 August 2021}

Published online: 26 August 2021

\section{References}

1. Lagergren J, Smyth E, Cunningham D, Lagergren P. Oesophageal cancer. Lancet. 2017;390:2383-96.

2. Cui Y, Chen H, Xi R, Cui H, Zhao Y, Xu E, et al. Whole-genome sequencing of 508 patients identifies key molecular features associated with poor prognosis in esophageal squamous cell carcinoma. Cell Res. 2020;30:902-13.

3. Koppula P, Zhuang L, Gan BJP. Cell: cystine transporter SLC7A11/xCT in cancer: ferroptosis, nutrient dependency, and cancer therapy. Protein Cell. 2020. https://doi.org/10.1007/s13238-020-00789-5.

4. Badgley MA, Kremer DM, Maurer HC, DelGiorno KE, Lee HJ, Purohit V, et al. Cysteine depletion induces pancreatic tumor ferroptosis in mice. Science. 2020;368:85-9.

5. Takeuchi S, Wada K, Toyooka T, Shinomiya N, Shimazaki H, Nakanishi $\mathrm{K}$, et al. Increased xCT expression correlates with tumor invasion and outcome in patients with glioblastomas. Neurosurgery. 2013;72:33-41 (discussion 41).

6. Lien EC, Ghisolfi L, Geck RC, Asara JM, Toker A. Oncogenic PI3K promotes methionine dependency in breast cancer cells through the cystineglutamate antiporter XCT. Sci Signal. 2017;10:eaao6604.

7. Shiozaki A, litaka D, Ichikawa D, Nakashima S, Fujiwara H, Okamoto K, et al. xCT, component of cysteine/glutamate transporter, as an independent prognostic factor in human esophageal squamous cell carcinoma. J Gastroenterol. 2014:49:853-63.

8. Okuno S, Sato H, Kuriyama-Matsumura K, Tamba M, Wang H, Sohda $\mathrm{S}$, et al. Role of cystine transport in intracellular glutathione level and cisplatin resistance in human ovarian cancer cell lines. Br J Cancer. 2003:88:951-6.

9. Dixon S, Lemberg K, Lamprecht M, Skouta R, Zaitsev E, Gleason C, et al. Ferroptosis: an iron-dependent form of nonapoptotic cell death. Cell. 2012;149:1060-72.

10. Lei G, Zhang Y, Koppula P, Liu X, Zhang J, Lin S, et al. The role of ferroptosis in ionizing radiation-induced cell death and tumor suppression. Cell Res. 2020;30:146-62.

11. Konieczkowski D, Johannessen C, Garraway LA. A convergence-based framework for cancer drug resistance. Cancer Cell. 2018;33:801-15.

12. Koppula P, Zhuang L, Gan B. Cystine transporter SLC7A11/xCT in cancer: ferroptosis, nutrient dependency, and cancer therapy. Protein Cell. 2020. https://doi.org/10.1007/s13238-020-00789-5.

13. Rojo de la Vega M, Chapman E, Zhang DD. NRF2 and the hallmarks of cancer. Cancer Cell. 2018;34:21-43.

14. Kawasaki Y, Okumura H, Uchikado Y, Kita Y, Sasaki K, Owaki T, et al. Nrf2 is useful for predicting the effect of chemoradiation therapy on esophageal squamous cell carcinoma. Ann Surg Oncol. 2014;21:2347-52.

15. Zhang G, Wang W, Yao C, Ren J, Zhang S, Han M. Salinomycin overcomes radioresistance in nasopharyngeal carcinoma cells by inhibiting Nrf2 level and promoting ROS generation. Biomed Pharmacother. 2017:91:147-54.

16. Xia D, Zhang XR, Ma YL, Zhao ZJ, Zhao R, Wang YY. Nrf2 promotes esophageal squamous cell carcinoma (ESCC) resistance to radiotherapy through the CaMKIla-associated activation of autophagy. Cell Biosci. 2020;10:90.
17. Shibata T, Ohta T, Tong K, Kokubu A, Odogawa R, Tsuta K, et al. Cancer related mutations in NRF2 impair its recognition by Keap1-Cul3 E3 ligase and promote malignancy. Proc Natl Acad Sci USA. 2008;105:13568-73.

18. Takahashi N, Cho P, Selfors LM, Kuiken HJ, Kaul R, Fujiwara T, et al. 3D culture models with CRISPR screens reveal hyperactive NRF2 as a prerequisite for spheroid formation via regulation of proliferation and ferroptosis. Mol Cell. 2020;80:828-844.e6.

19. Fan Z, Wirth AK, Chen D, Wruck CJ, Rauh M, Buchfelder M, et al. Nrf2Keap1 pathway promotes cell proliferation and diminishes ferroptosis. Oncogenesis. 2017;6:e371.

20. Lee YJ, Kim WI, Bae JH, Cho MK, Lee SH, Nam HS, et al. Overexpression of Nrf2 promotes colon cancer progression via ERK and AKT signaling pathways. Ann Surg Treat Res. 2020;98:159-67.

21. Ma Z, Zhang H, Lian M, Yue C, Dong G, Jin Y, et al. SLC7A11, a component of cysteine/glutamate transporter, is a novel biomarker for the diagnosis and prognosis in laryngeal squamous cell carcinoma. Oncol Rep. 2017;38:3019-29.

22. Tian J, Wei X, Zhang W, Xu A. Effects of selenium nanoparticles combined with radiotherapy on lung cancer cells. Front Bioeng Biotechnol. 2020:8:598997.

23. Dai F, Lee H, Zhang Y, Zhuang L, Yao H, Xi Y, et al. BAP1 inhibits the ER stress gene regulatory network and modulates metabolic stress response. Proc Natl Acad Sci USA. 2017;114:3192-7.

24. Zhang Y, Shi J, Liu X, Feng L, Gong Z, Koppula P, et al. BAP1 links metabolic regulation of ferroptosis to tumour suppression. Nat Cell Biol. 2018;20:1181-92.

25. Yang H, Do H, Kim D, Park J, Chang W, Chung H, et al. Transcriptional regulation of human Oct4 by steroidogenic factor-1. J Cell Biochem. 2007;101:1198-209.

26. Li X, Liu C, Zhu Y, Rao H, Liu M, Gui L, et al. SETD2 epidermal deficiency promotes cutaneous wound healing via activation of AKT/mTOR Signalling. Cell Prolif. 2021;54:e13045.

27. Fornes $\mathrm{O}$, Castro-Mondragon JA, Khan $\mathrm{A}$, van der Lee $\mathrm{R}$, Zhang $\mathrm{X}$, Richmond PA, et al. JASPAR 2020: update of the open-access database of transcription factor binding profiles. Nucleic Acids Res. 2020;48:D87-92.

28. Ferino A, Rapozzi V, Xodo LE. The ROS-KRAS-Nrf2 axis in the control of the redox homeostasis and the intersection with survival-apoptosis pathways: Implications for photodynamic therapy. J Photochem Photobiol B. 2020;202:111672

29. Li B, Yang L, Peng X, Fan Q, Wei S, Yang S, et al. Emerging mechanisms and applications of ferroptosis in the treatment of resistant cancers. Biomed Pharmacother. 2020;130:110710.

30. Xie Y, Hou W, Song X, Yu Y, Huang J, Sun X, et al. Ferroptosis: process and function. Cell Death Differ. 2016;23:369-79.

31. Park E, Chung SW. ROS-mediated autophagy increases intracellular iron levels and ferroptosis by ferritin and transferrin receptor regulation. Cell Death Dis. 2019;10:822.

32. Stockwell BR, Friedmann Angeli JP, Bayir H, Bush Al, Conrad M, Dixon SJ, et al. Ferroptosis: a regulated cell death nexus linking metabolism, redox biology, and disease. Cell. 2017;171:273-85.

33. Yang M, Chen P, Liu J, Zhu S, Kroemer G, Klionsky DJ, et al. Clockophagy is a novel selective autophagy process favoring ferroptosis. Sci Adv. 2019.5:eaaw2238.

34. Zhou S, Ye W, Shao Q, Zhang M, Liang JJ. Nrf2 is a potential therapeutic target in radioresistance in human cancer. Crit Rev Oncol Hematol. 2013;88:706-15.

35. Zhao Q, Mao A, Yan J, Sun C, Di C, Zhou X, et al. Downregulation of Nrf2 promotes radiation-induced apoptosis through $\mathrm{Nrf2}$ mediated Notch signaling in non-small cell lung cancer cells. Int J Oncol. 2016;48:765-73.

36. Shibata T, Kokubu A, Saito S, Narisawa-Saito M, Sasaki H, Aoyagi K, et al. NRF2 mutation confers malignant potential and resistance to chemoradiation therapy in advanced esophageal squamous cancer. Neoplasia. 2011;13:864-73.

37. Lang X, Green MD, Wang W, Yu J, Choi JE, Jiang L, et al. Radiotherapy and immunotherapy promote tumoral lipid oxidation and ferroptosis via synergistic repression of SLC7A11. Cancer Discov. 2019;9:1673-85.

38. Lin W, Wang C, Liu G, Bi C, Wang X, Zhou Q, et al. SLC7A11/xCT in cancer: biological functions and therapeutic implications. Am J Cancer Res. 2020;10:3106-26. 
39. Sun X, Ou Z, Chen R, Niu X, Chen D, Kang R, et al. Activation of the p62-Keap1-NRF2 pathway protects against ferroptosis in hepatocellular carcinoma cells. Hepatology. 2016;63:173-84.

40. Dodson M, Castro-Portuguez R, Zhang DD. NRF2 plays a critical role in mitigating lipid peroxidation and ferroptosis. Redox Biol. 2019;23:101107.

41. La Rosa P, Petrillo S, Turchi R, Berardinelli F, Schirinzi T, Vasco G, et al. The Nrf2 induction prevents ferroptosis in Friedreich's Ataxia. Redox Biol. 2021;38:101791.

42. Chen D, Tavana O, Chu B, Erber L, Chen Y, Baer R, et al. NRF2 Is a major target of ARF in p53-independent tumor suppression. Mol Cell. 2017;68:224232.e4.

43. Liu Q, Gao Y, Ci X. Role of Nrf2 and its activators in respiratory diseases. Oxid Med Cell Longev. 2019;2019:7090534.

44. Jeong Y, Hoang NT, Lovejoy A, Stehr H, Newman AM, Gentles AJ, et al. Role of KEAP1/NRF2 and TP53 mutations in lung squamous cell carcinoma development and radiation resistance. Cancer Discov. 2017;7:86-101.

45. Satoh H, Moriguchi T, Takai J, Ebina M, Yamamoto M. Nrf2 prevents initiation but accelerates progression through the Kras signaling pathway during lung carcinogenesis. Cancer Res. 2013;73:4158-68.

46. Tao S, Rojo de la Vega M, Chapman E, Ooi A, Zhang DD. The effects of NRF2 modulation on the initiation and progression of chemically and genetically induced lung cancer. Mol Carcinog. 2018;57:182-92.

47. Qiang Z, Dong H, Xia Y, Chai D, Hu R, Jiang H. Nrf2 and STAT3 alleviates ferroptosis-mediated IIR-ALI by regulating SLC7A11. Oxid Med Cell Longev. 2020;2020:5146982

48. Lee YS, Lee DH, Choudry HA, Bartlett DL, Lee YJ. Ferroptosis-induced endoplasmic reticulum stress: cross-talk between ferroptosis and apoptosis. Mol Cancer Res. 2018;16:1073-6.

49. Kerins MJ, Ooi A. The roles of NRF2 in modulating cellular iron homeostasis. Antioxid Redox Signal. 2018;29:1756-73.

50. Sui X, Zhang R, Liu S, Duan T, Zhai L, Zhang M, et al. RSL3 drives ferroptosis through GPX4 inactivation and ROS production in colorectal cancer. Front Pharmacol. 2018;9:1371.

51. Shin D, Kim EH, Lee J, Roh JL. Nrf2 inhibition reverses resistance to GPX4 inhibitor-induced ferroptosis in head and neck cancer. Free Radic Biol Med. 2018;129:454-62.

52. Roh JL, Kim EH, Jang H, Shin D. Nrf2 inhibition reverses the resistance of cisplatin-resistant head and neck cancer cells to artesunate-induced ferroptosis. Redox Biol. 2017;11:254-62.

53. Gai C, Yu M, Li Z, Wang Y, Ding D, Zheng J, et al. Acetaminophen sensitizing erastin-induced ferroptosis via modulation of Nrf2/heme oxygenase-1 signaling pathway in non-small-cell lung cancer. J Cell Physiol. 2020;235:3329-39.

54. Xue D, Zhou X, Qiu J. Emerging role of NRF2 in ROS-mediated tumor chemoresistance. Biomed Pharmacother. 2020;131:110676.
55. Su $\sqcup$, Zhang JH, Gomez H, Murugan R, Hong X, Xu D, et al. Reactive oxygen species-induced lipid peroxidation in apoptosis, autophagy, and ferroptosis. Oxid Med Cell Longev. 2019;2019:5080843.

56. Lei G, Mao C, Yan Y, Zhuang L, Gan B. Ferroptosis, radiotherapy, and combination therapeutic strategies. Protein Cell. 2021. https://doi.org/10. 1007/s13238-021-00841-y.

57. Yang J, Zhou Y, Xie S, Wang J, Li Z, Chen L, et al. Metformin induces ferroptosis by inhibiting UFMylation of SLC7A11 in breast cancer. J Exp Clin Cancer Res. 2021;40:206.

58. Zhao X, Gao M, Liang J, Chen Y, Wang Y, Wang Y, et al. SLC7A11 reduces laser-induced choroidal neovascularization by inhibiting RPE ferroptosis and VEGF production. Front Cell Dev Biol. 2021;9:639851.

59. Cobler L, Zhang H, Suri P, Park C, Timmerman LA. xCT inhibition sensitizes tumors to $\gamma$-radiation via glutathione reduction. Oncotarget. 2018;9:32280-97.

60. Nagane M, Kanai E, Shibata Y, Shimizu T, Yoshioka C, Maruo T, et al. Sulfasalazine, an inhibitor of the cystine-glutamate antiporter, reduces DNA damage repair and enhances radiosensitivity in murine B16F10 melanoma. PLoS ONE. 2018;13:e0195151.

61. Carpi-Santos R, Calaza KC. Alterations in system $\times(c)(-)$ expression in the retina of Type 1 diabetic rats and the role of Nrf2. Mol Neurobiol. 2018;55:7941-8.

62. Silva-Adaya D, Ramos-Chávez LA, Petrosyan P, González-Alfonso WL, Pérez-Acosta A, Gonsebatt ME. Early neurotoxic effects of inorganic arsenic modulate cortical GSH levels associated with the activation of the Nrf2 and NFKB pathways, expression of amino acid transporters and NMDA receptors and the production of hydrogen sulfide. Front Cell Neurosci. 2020;14:17.

63. Ono K, Furugen A, Kurosawa Y, Jinno N, Narumi K, Kobayashi M, et al. Analysis of the effects of polyunsaturated fatty acids on transporter expressions using a PCR array: induction of XCT/SLC7A1 1 in human placental BeWo cells. Placenta. 2019;75:34-41.

64. Zhang L, Huang Y, Ling J, Zhuo W, Yu Z, Luo Y, et al. Overexpression of SLC7A11: a novel oncogene and an indicator of unfavorable prognosis for liver carcinoma. Future Oncol. 2018;14:927-36.

65. Zhong W, Weiss HL, Jayswal RD, Hensley PJ, Downes LM, St Clair DK, et al. Extracellular redox state shift: a novel approach to target prostate cancer invasion. Free Radic Biol Med. 2018:117:99-109.

\section{Publisher's Note}

Springer Nature remains neutral with regard to jurisdictional claims in published maps and institutional affiliations.

Ready to submit your research? Choose BMC and benefit from:

- fast, convenient online submission

- thorough peer review by experienced researchers in your field

- rapid publication on acceptance

- support for research data, including large and complex data types

- gold Open Access which fosters wider collaboration and increased citations

- maximum visibility for your research: over 100M website views per year

At BMC, research is always in progress.

Learn more biomedcentral.com/submissions 